\title{
Referees for 2017
}

(C) Springer Science+Business Media B.V., part of Springer Nature 2017

The Journal of Ethics thanks both the members of its distinguished editorial board and the following philosophers for their excellent assistance to the journal by way of their service as referees:

\author{
Skott Brill \\ Tom Buller \\ John Dreher \\ Rachel Fernflores \\ Ishtiyaque Haji \\ Donald C. Hubin \\ Jens Johansson \\ Donald Marquis \\ Brian Powell \\ Rosamond Rhodes \\ Mark Schroeder \\ Re'em Segev \\ Eric Vogelstein \\ Michael J. Zimmerman
}

